\title{
Sunitinib Induced Diffuse Alveolar Hemorrhage: A Case Report
}

\author{
Supreet Sethi ${ }^{\mathrm{a}}$, Nutan Bhaskar ${ }^{\mathrm{b}, \mathrm{c}}$
}

\begin{abstract}
A 68-year-old man with papillary renal cell carcinoma of the transplanted kidney presented with worsening respiratory distress while on treatment with sunitinib. Computerized tomography of chest revealed diffuse ground-glass opacities, and sequentially bloody bronchoalveolar lavage fluid was noted on bronchoscopy. There was marked clinico-radiologic improvement after withdrawal of sunitinib. This is the first case report of diffuse alveolar hemorrhage as an adverse effect of sunitinib in non-metastatic papillary renal cell carcinoma. With the increasing use of sunitinib in treatment of solid tumors, it is important to be aware of this potential adverse effect. Clinicians need to be vigilant for alveolar hemorrhage in patients on sunitinib with or without evidence of lung metastasis.
\end{abstract}

Keywords: Diffuse Alveolar Hemorrhage; Tyrosine kinase inhibitor; Renal cell carcinoma; Respiratory failure

\section{Introduction}

Sunitinib malate is a multitargeted receptor tyrosine kinase inhibitor, with a high oral bioavailability, has been approved by FDA for use in metastatic renal cell carcinoma (RCC) and gastrointestinal stromal tumor (GIST) that are refractory to first line treatment with Imatinib. It is usually well tolerated with mild-moderate side effects. As with any other chemo-

Manuscript accepted for publication January 2, 2013

\footnotetext{
${ }^{a}$ Department of Internal Medicine, Division of Pulmonary and Critical Care Medicine, University of Arkansas for Medical sciences, 4301 West Markham Street, Little Rock, AR, USA

${ }^{\mathrm{b}}$ University of Arkansas for Medical Sciences, USA

${ }^{\mathrm{c}}$ Corresponding author: Nutan Bhaskar, University of Arkansas for the Medical Sciences, 4301 West Markham Street, Slot \#555, Little Rock, AR, USA. Email: nbhaskar@uams.edu
}

doi: http://dx.doi.org/10.4021/jmc1011w therapeutic agent, the effect is seen not only in the malignant clone of cells but also in the healthy cells leading to many of its adverse effects. As we continue to learn more about this novel agent, rare and some serious side effects are being reported. We hereby describe a case of alveolar hemorrhage secondary to sunitinib use for a patient with advanced renal cell carcinoma of allograft kidney with no evidence of metastatic disease to lungs.

\section{Case Report}

A 68-year-old man with end stage renal disease secondary to diabetes mellitus who received a deceased donor kidney transplant four years ago was diagnosed with papillary carcinoma of the transplanted kidney, stage T3a N1M0 according to TNM staging for renal carcinoma with retroperitoneal lymph node involvement. He underwent an allograft nephrectomy and was subsequently started on sunitinib at a dose of $25 \mathrm{mg}$ daily on a 4 weeks on; 2 weeks off schedule. He tolerated it well with only mild nausea. Six months into his treatment with sunitinib, patient presented to the hospital with acute onset dyspnea of one day duration. He denied of fevers, productive cough or chest pain.

Examination revealed the patient to be in moderate re-

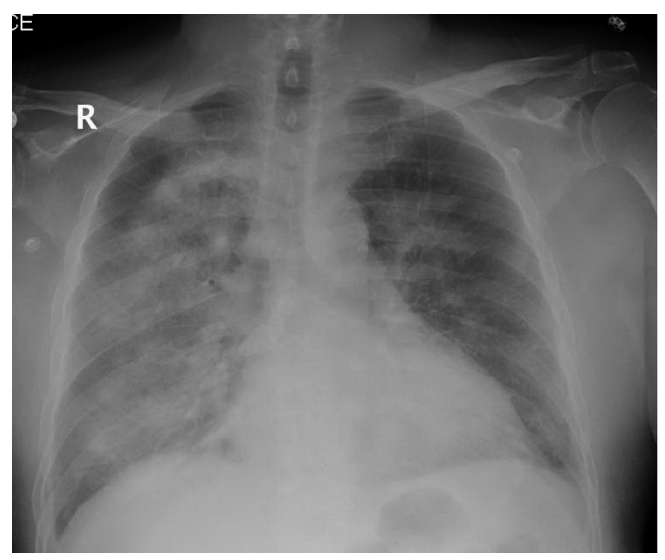

Figure 1. Chest radiograph showing diffuse bilateral alveolar opacities. 


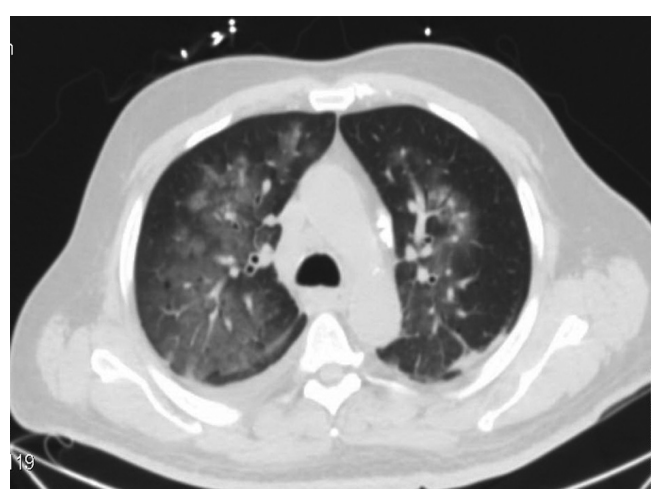

Figure 2. Computerized tomographic images of the chest with fluffy bilateral ground glass opacities.

spiratory distress. His pulse was 100 beats/minute, mean arterial blood pressure was $80 \mathrm{mmHg}$, respiratory rate of 30/ minute and oxygen saturation was $90 \%$ on six liters nasal cannula. There was no jugular venous distension. Auscultation revealed audible S1 and S2 without murmurs and coarse crackles in bilateral lung fields. Abdomen was nontender without rebound or guarding and no hepatosplenomegaly was appreciated. Mild pitting edema of lower extremities was noted.

Laboratory data revealed a white count of $8,400 / \mathrm{mm}^{3}$, hemoglobin of $9 \mathrm{~g} / \mathrm{dL}$, platelet count of $147,000 / \mathrm{mm}^{3}$ and normal serum chemistries. Arterial blood analysis revealed a $\mathrm{pH}$ of 7.36, arterial oxygen tension, $64 \mathrm{mmHg}, \mathrm{Co} 2$ tension, $38 \mathrm{mmHg}$. Chest radiograph showed diffuse bilateral alveolar opacities (Fig. 1). Computerized tomographic images of the chest revealed bilateral ground glass alveolar opacities (Fig. 2).

Patient was dialyzed for presumed fluid overload and was also started on broad spectrum antibiotics for possible pneumonia. Dialysis with ultra-filtration resulted in modest clinical improvement but the chest radiograph continued to show bilateral alveolar opacities. Blood cultures remained negative. The next day patient had an unexplained drop in hemoglobin from $9.0 \mathrm{~g} / \mathrm{dL}$ to $6.3 \mathrm{~g} / \mathrm{dL}$. Flexible bronchoscopy was performed which revealed persistently bloody returns on sequential broncho-alveolar lavage (BAL), consistent with diffuse alveolar hemorrhage and the cultures and cytology remained negative. Sunitinib was held which resulted in marked clinical and radiographic improvement (Fig. 3). Patient was weaned off supplemental oxygen. Sunitinib was withheld and alternative chemotherapy regimen was considered.

\section{Discussion}

Sunitinib malate is a multitargeted receptor tyrosine kinase inhibitor, which is usually well tolerated. Its effect is

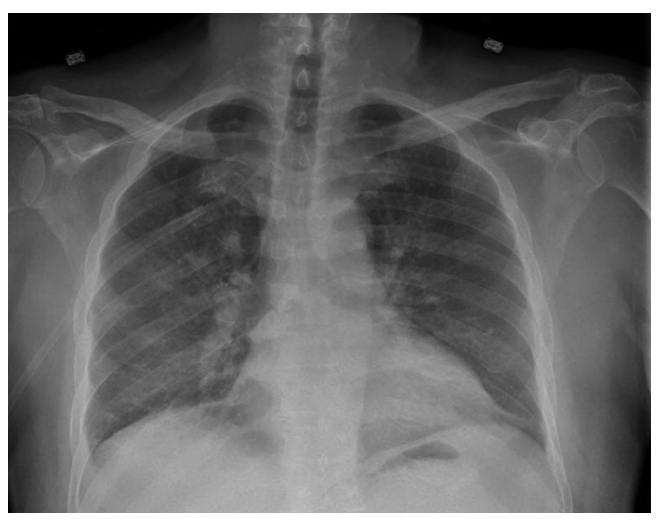

Figure 3. Follow-up chest radiograph showing marked radiographic improvement and resolution of previously noted opacities.

seen not only in the malignant clone of cells but also in the healthy cells leading to many of its adverse effects. Tyrosine kinase is a key enzyme utilized in many intracellular signaling pathways. Sunitinib targets receptors for platelet derived growth factors $\alpha$ and $\beta$ and vascular endothelial growth factors (VEGF) [1]. The inhibition of these receptors plays a key role in reducing tumor angiogenesis, causing cell death and shrinkage of the tumor [2]. It also inhibits other receptor tyrosine kinases including stem cell factor receptor, FMSlike tyrosine kinase-3 receptor, colony stimulating factor-1 receptor and glial cell line derived neurotrophic factor receptor. Due to its action on multiple receptors, a substantial clinical effect has been seen not only in metastatic renal cell carcinoma and GIST, but also in non-small cell lung cancer and other tumors [3]. The adverse effects noted with sunitinib are due to its multi-receptor action affecting not only malignant tissues but healthy tissues as well. These include fatigue, diarrhea, anorexia, hematotoxicity, stomatitis, hand- foot syndrome, nausea, skin problems, hypothyroidism, bleeding and abnormal heart function. Rare and serious adverse effects have been reported in isolated case reports. These include bowel perforation, leukocytoclastic vasculitis, hyperparathyroidism, and gastrointestinal hemorrhage in patients treated for GIST) [4-6]. There has been only one case report of diffuse alveolar hemorrhage secondary to sunitinib in a patient with renal cell carcinoma metastatic to lung [7].

Alveolar hemorrhage has been reported as an adverse effect with the development of anti-angiogenic therapeutic agents. Fatal tumor-related bleeding in patients treated with bevacizumab, an anti-angiogenic, recombinant humanized monoclonal antibody that binds to VEGF is well known [8]. Sunitinib led to fatal alveolar hemorrhage events in two patients with advanced squamous cell lung cancer in a phase II trial of previously treated advanced non-small cell lung cancer [9]. Our case is unique as the patient did not have any evidence of lung metastasis but developed alveolar hemorrhage while on sunitinib. Vascular endothelial growth factor 
is one of the principal pro-angiogenic factors in solid tumors and has a key role in endothelial cell growth and survival. VEGF coordinates proper development of the normal lung epithelium and vasculature. Inhibition of VEGF results in inhibition of angiogenesis and vasculogenesis, decrease in the regenerative capacity of the endothelial cells causing endothelial dysfunction in the supporting layers of the blood vessels. The inhibition of VEGF is a possible mechanism involved in the destruction of normal lung tissue and subsequent alveolar hemorrhage.

Although these targeted therapies have drastically improved the prognosis of patients with advanced RCC, there is still much to be learnt about their optimal dosing and scheduling. The recently concluded EFFECT trial revealed superiority of $4 / 2$ schedule over daily continuous dosing in regards to optimum therapeutic drug concentrations [9]. With the increasing use of sunitinib in treatment of solid tumors, it is important to be aware of this potential adverse effect. Physicians need to be vigilant for alveolar hemorrhage in patients on sunitinib with or without evidence of lung metastasis.

\section{Financial Disclosure}

No sources of funding were used in this study.

\section{Conflicts of Interest}

No potential conflicts of interest exist.

\section{Abbreviations}

GIST: gastrointestinal stromal tumor; RC: renal cell carcinoma; VEGF: vascular endothelial growth factors

\section{References}

1. Zhao L, Wang K, Ferrara N, Vu TH. Vascular endothelial growth factor co-ordinates proper development of lung epithelium and vasculature. Mech Dev. 2005;122(78):877-886.

2. Folkman J. Tumor angiogenesis: therapeutic implications. N Engl J Med. 1971;285(21):1182-1186.

3. Socinski MA. The current status and evolving role of sunitinib in non-small cell lung cancer. J Thorac Oncol. 2008;3(6 Suppl 2):S119-123.

4. Karadimou A, Migou M, Economidi A, Stratigos A, Kittas C, Dimopoulos MA, Bamias A. Leukocytoclastic vasculitis after long-term treatment with sunitinib: a case report. Case Rep Oncol. 2011;4(2):385-391.

5. Hoshino Y, Hasegawa H, Ishii Y, Endo T, Ochiai H, Okabayashi K, Kaneko G, et al. Two cases of bowel perforation associated with sunitinib treatment for renal cell carcinoma. Int J Clin Oncol. 2012;17(4):412-416.

6. Baldazzi V, Tassi R, Lapini A, Lunghi A, Garofoli E, Caruso S, Carini M, et al. Sunitinib-induced hyperparathyroidism: a possible mechanism to altered bone homeostasis. Cancer. 2012;118(12):3165-3172.

7. Yamada T, Ohtsubo K, Izumi K, Takeuchi S, Mouri H, Yamashita K, Yasumoto K, et al. Metastatic renal cell carcinoma complicated with diffuse alveolar hemorrhage: a rare adverse effect of sunitinib. Int J Clin Oncol. 2010;15(6):638-641.

8. Cho YJ, Murgu SD, Colt HG. Bronchoscopy for bevacizumab-related hemoptysis. Lung Cancer. 2007;56(3):465-468.

9. Motzer RJ, Hutson TE, Olsen MR, Hudes GR, Burke JM, Edenfield WJ, Wilding G, et al. Randomized phase II trial of sunitinib on an intermittent versus continuous dosing schedule as first-line therapy for advanced renal cell carcinoma. J Clin Oncol. 2012;30(12):1371-1377. 